\title{
A Comparison Study of Cervical Flexion-Relaxation Ratio in the Normal and Forward Head Postures
}

\author{
Sang-Seok Yeo, Jung-Won Kwon \\ Department of Physical Therapy, College of Health Sciences, Dankook University, Cheonan, Republic of Korea
}

Purpose: This study aimed to examine the effects of forward head posture on the flexion-relaxation ratio (FRR) and muscle activity during sustained neck flexion and to investigate the correlation between craniovertebral angle and FRR.

Methods: Nineteen subjects participated in this study and were allocated to a forward head posture (FHP) group or a non-forward head posture (NFHP) group. Craniovertebral angle (CVA) and FRR were measured in all subjects, and all participants performed a standardized cervical flexion-extension movement in two phases: Phase I, sustained cervical full flexion for $5 \mathrm{~s}$; and Phase II, cervical extension with the return to the starting position for $5 \mathrm{~s}$. The value of CVA has calculated three times, and the value of FRR was measured three times in order to obtain the mean value.

Results: FRR values in the FHP and NFHP group were significantly different $(p<0.05)$. Phase I was significantly different, but the Phase II was not significantly different between the FHP and NFHP group $(p>0.05)$. There was a significant correlation between the muscle activity of Phase I and CVA $(p<0.05)$. However, FRR and the muscle activity of the Phase II were not a significant correlation with the CVA $(p>0.05)$.

Conclusion: FHP increases the muscle activity of the cervical erector spinae during sustained neck flexion and reduces FRR, which can cause fatigue in the cervical erector spinae. In addition, for those with a smaller CVA, muscle activity of the cervical erector spinae is increased during sustained neck flexion, which can increase neck muscle tension.

Keywords: Forward head posture, Flexion-relaxation ratio, Craniovertebral angle

\section{INTRODUCTION}

Forward Head Posture (FHP) is a poor habitual neck posture called iHunch and is defined as hypertension of upper cervical vertebrae and forward translation of cervical vertebrae. ${ }^{1}$ Due to frequent computer work and smartphone use, upper trapezius and neck muscles are repeatedly overused, which results in muscle fiber damage, cumulative damage from acute trauma, and muscle fatigue commonly in the neck and shoulders. ${ }^{2-4}$ Repeated damage causes FHP due to weakening of lower cervical and upper thoracic erector spinae and scapular retractor muscles, and capital flexors. Cervical spinae and head attached to the scapula and upper thorax and suboccipital region muscles shortened relative to normal posture, causing secondary problems such as cervicogenic headache and referred pain. $^{5}$
FHP can place stresses on muscles and connective tissue in the cervical spine region. ${ }^{6}$ As occurs in other postural and movement dysfunctions, FHP often results in movement compensations due to regional musculature becoming excessively underactive or overactive. ${ }^{7}$ Individuals with FHP underuse certain muscles such as deep neck flexors, rhomboids, lower trapezius, and cervical erector spinae, and underactivity in these muscles makes it difficult for the cervical spine to maintain an upright position. ${ }^{8}$ Advanced stages of FHP can contribute to upper cervical spine compression, which reduces the ability of the first cervical vertebrae $(\mathrm{Cl})$ to rotate around the second vertebrae (C2) and can result in increased risk of spinal instability, degeneration, and pain. ${ }^{9}$

In a study to quantitatively evaluate these pain and determine therapeutic effects, Murphy et al. mentioned the Flexion-Relaxation Phenomenon (FRP). ${ }^{10}$ Callaghan and Dunk ${ }^{11}$ reported the FRP as a decrease or Attribution Non-commercial License (https:// creativecommons.org/license/by-nc/4.o.) which permits unrestricted non-commercial use, distribution, and reproduction in any medium, provided the original work is properly cited. 
sudden onset of myoelectric "silence" in erector spinae during trunk flexion. FRP, in response to passive tissue that can sustain the moment placed on the joint, refers to the mechanism of loss of muscle activity in the range of motion. ${ }^{11}$ Compared to normal people, subjects with damaged neuromuscular systems reported loss of flexion-relaxation phenomenon and ratio (FRR) in the end range of motion. ${ }^{12,13}$ The calculation method is determined by dividing the maximum activity measured during these movements by the average muscle activity measured during the fully flexed position. ${ }^{10,14}$ Specifically, extension of the paravertebral muscles provides mechanical stimulation to the structure around the neck joint, and a stress-suppression reaction takes place to provide stability within the end range of the structure. ${ }^{15}$ FRP can be presented to the FRR by measuring the cervical erector muscles during flexion. ${ }^{16}$ The FRR has been commonly used to measure the relaxation capacity of the muscles of the neck. Several studies have addressed FRP in patients with lower back pain. ${ }^{13,17}$ Furthermore, cervical FRP is similar to lumbar joint FRP, and recent studies have suggested FRR may be an important marker of neuromuscular impairment. ${ }^{18}$ A previous study reported that cervical FRR was significantly lower for neck pain patients than healthy subjects, ${ }^{10}$ and another reported FRR might be used as a tool to identify potential risk factors for neck joints. ${ }^{19}$

Studies have addressed the association between FHP and articular angle and muscle activity, but few studies have investigated FRR in subjects with FHP. The purpose of this study was to compare FRR in the presence or absence of FHP and to investigate muscle activity differences during sustained neck flexion.

\section{METHODS}

\section{Subjects}

A total of 24 adults were recruited for this study. Of them, 19 participants completed this study. There were five drop-outs in both groups. Three Table 1. General characteristics of the subjects

\begin{tabular}{lccc}
\hline Group & FHP & NFHP & p \\
\hline Gender (M/F) & $2 / 8$ & $5 / 4$ & 0.808 \\
Height $(\mathrm{cm})$ & $165.3 \pm 6.6$ & $169.3 \pm 7.5$ & 0.260 \\
Age $(\mathrm{yr})$ & $21.6 \pm 1.2$ & $21.6 \pm 2.2$ & 0.847 \\
Weight $(\mathrm{kg})$ & $57.1 \pm 12.9$ & $62.6 \pm 8.4$ & 0.315 \\
CVA $\left(^{\circ}\right)$ & $46.7 \pm 3.6$ & $57.5 \pm 4.5$ & $<0.001^{*}$ \\
\hline
\end{tabular}

Mean \pm SD

FHP: forward head posture, NFHP: non-forward head posture, CVA: craniovertebral angle.

${ }^{*} p<0.05$. participants had medical conditions or problems that might make testing unreliable. Two participants had problems with electromyogram data. Thus, they were excluded. A total of 19 subjects ( 7 men and 12 women with ages ranging from 20 to 29 years) were voluntarily recruited at a local university. Subjects were allocated to an FHP group when the angle between the horizontal line of the $\mathrm{C} 7$ spinous process (defined as the craniovertebral angle (CVA) and the tragus was $<48.7$ degrees or to a Non-Forward Head Posture (NFHP) group when the angle was $\geq 48.7$ degrees. ${ }^{14}$ Inclusion criteria were as follows: 1) no treatment for FHP within the previous 3 months, 2) no history of musculoskeletal or neurologic disease, 3) no pain in the neck or shoulders, and 4) no surgery within the previous 3 months. All subjects were provided an overview of the purpose of the study and the procedures involved and provided written consent. Demographic data was collected and it was not significantly different in the FHP and NFHP groups $(\mathrm{p}>0.05)$ (Table 1).

\section{Measurements}

\section{1) Craniovertebral angle}

A camera fitted with a tripod was fixed on a flat table at $80 \mathrm{~cm}$ from the face of the subject, who stood with profile toward the camera. The camera was positioned at the level of the C7 vertebrae (Figure 1). CVA was measured between a horizontal line at the $\mathrm{C} 7$ spinous process and the tragus three times using the GNU Image Manipulation Program ver. 2.10.12 (Spencer Kimball, Peter Mattis. CA, USA). The value of CVA was calculated three times in order to obtain the mean value.

\section{2) Electromyography}

Muscle activity was measured by surface electromyography (EMG) Telemyo DTS Desk RECEIVER (Noraxon, Scottsdale, USA). EMG signal data were sampled at $1,000 \mathrm{~Hz}$, bandpass filtered between 10 and $500 \mathrm{~Hz}$, and

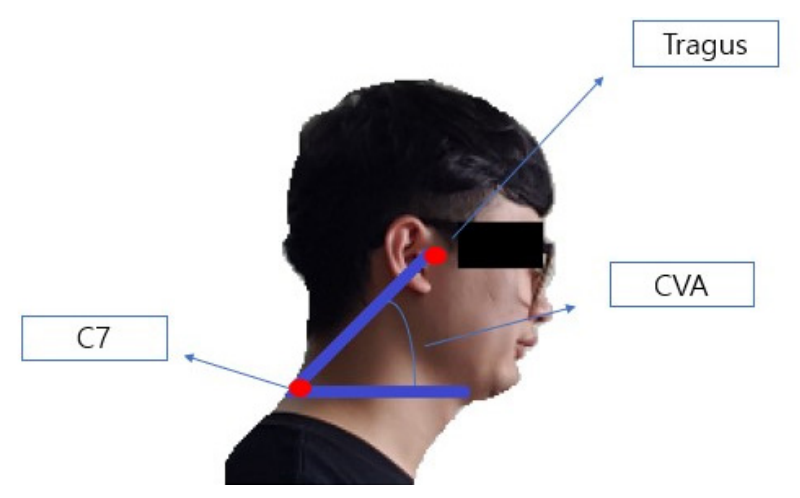

Figure 1. Craniovertebral angle (CVA) measurement. 
converted to digital signals using MR3 software (Noraxon, Scottsdale, USA). A notch filter at $60 \mathrm{~Hz}$ was employed to clean power line noise. The data were further processed in the electromyographic silence during full flexion and during neck extension by measuring the root mean square (RMS) on a 50 ms window of time. EMG electrodes were placed bilaterally $2 \mathrm{~cm}$ from midline at the level of the $\mathrm{C} 4$ spinous process, longitudinally in the direction of muscle fibers to record activity of the cervical erector spinae. A reference electrode was taped to the right wrist. The electrodes were placed as previously described..$^{20}$ All participants performed a standardized cervical flexion-extension movement in two phases: Phase I, sustained cervical full flexion for 5 seconds; and Phase II, cervical extension with return to the starting position for 5 seconds. The FRR was measured three times in order to obtain the mean value.

\section{Flexion-relaxation ratio}

Subjects were asked to perform a standardized cervical flexion-extension movement in two phases: phase I, sustained cervical full flexion for $5 \mathrm{sec}-$ onds; and phase II, cervical extension followed by return to the starting position for 5 seconds. ${ }^{21}$ Motion speeds were standardized using a metronome to prevent motion speed affecting FRP during measurements. Subjects performed two familiarization procedures and three trials at intervals of 60 seconds. FRR was calculated by dividing maximum muscle activity during the 5 seconds of phase II by average activation during phase I. ${ }^{14}$

$$
\mathrm{FRR}=\frac{\text { Maximum EMG in Phase } \mathbb{I}}{\text { Average EMG in Phase I }}
$$

\section{Statistical analysis}

General subject characteristics were analyzed using descriptive statistics. Shapiro-Wilk test was used for normality test among the outcome measures. The independent t-test was used to determine the significances of age, height, weight, and CVA differences between the FHP and NFHP groups. The independent t-test was used to compare the FRR values, and Pearson correlation analysis was used to determine the association between CVA and FRR. SPSS software ver. 20.0 (IBM Corp., NY, USA) was used for the data analysis. Null hypotheses of no difference were rejected when p-values were $<0.05$.

\section{RESULTS}

No significant intergroup difference was observed for age, height, or weight. However, mean FHP angles were significantly different in the two groups $(\mathrm{p}<0.05)$ (Table 1). Muscle activities and FRR values of cervical erector spinae are summarized in Table 2 . FRR values were significantly different in the FHP and NFHP groups ( $\mathrm{p}<0.05)$. Muscle activity of Phase I was significantly different, but muscle activity of Phase II was not significantly different between the FHP and NFHP groups ( $\mathrm{p}>0.05$ ). Correlation analysis showed that there was a significantly negative correlation between the muscle activity of Phase I and CVA $(\mathrm{p}<0.05)$. However, FRR and the muscle activity of Phase II were not significantly correlated with the CVA $(\mathrm{p}>0.05)$.

\section{DISCUSSION}

In the current study, we investigated the effects of FHP and NFHP group on FRR during sustained neck flexion. FRR was significantly lower in the FHP group than in the NFHP group, and muscle activity of the Phase I in the FHP group was lower than in the NFHP group. Correlation analysis showed that CVA was significantly negative correlation with the muscle activity of Phase I, which indicated that a smaller CVA angle made it more difficult to completely relax neck muscles.

Previous studies have reported that FHP increases flexion moment of the head by extending the neck, and thus, increases loads on tissues around the neck. Continuing this abnormal posture reduces the stability

Table 2. Comparison and correlation analysis for the CVA, FRR and each phase between FHP and NFHP group

\begin{tabular}{|c|c|c|c|c|c|c|}
\hline & \multirow{2}{*}{$\mathrm{FHP}$} & \multirow{2}{*}{ NFHP } & \multirow{2}{*}{$\mathrm{t}$} & \multirow{2}{*}{$\mathrm{p}$} & \multicolumn{2}{|c|}{ CVA } \\
\hline & & & & & Pearson $r$ & $\mathrm{p}$ \\
\hline FRR & $2.06 \pm 0.43$ & $4.67 \pm 2.80$ & -2.648 & $0.019 *$ & 0.389 & 0.123 \\
\hline Phase 1 ( $\mu v)$ & $50.21 \pm 14.97$ & $27.28 \pm 12.00$ & 3.504 & $0.003^{*}$ & -0.498 & $0.042^{*}$ \\
\hline Phase 2 ( $\mu \mathrm{v})$ & $98.81 \pm 11.87$ & $101.33 \pm 9.75$ & -0.480 & 0.683 & 0.280 & 0.916 \\
\hline
\end{tabular}

Mean \pm SD

FRR unit: \%RVC; FHP: forward head posture, NFHP: non-forward head posture, CVA: craniovertebral angle, FRR: flexion relaxation ratio, Phase 1: sustain cervical full flexion, Phase 2: extension with return to the starting position.

${ }^{*} p<0.05$. 
of viscous tissues and can change muscle length-tension properties. ${ }^{22}$ Since greater neck flexion angles are required to produce the same force in passive tissues, posterior neck muscles remain active over a wider range of flexion to control motion and provide cervical stability. ${ }^{23}$ Furthermore, it has been reported that cervical muscle hyperactivity caused by deformation reduces FRR. ${ }^{24}$ These loads on the cervical spine can induce tension and muscle fatigue and affect muscles and ligaments. Indahl et al. ${ }^{25}$ suggested that the complex regulatory mechanisms of reflexes control movements of spinal areas and suggested that loss of FRP might be due to imbalance between neural outputs from damaged structures to muscles, which can lead to additional muscle activity to protect spinal structures. Our findings are consistent with those of previous studies, which reported FHP patients had higher neck muscle activities during sustained full neck flexion. In addition, we believe FRR reductions observed in the FHP group were manifestations of a process that protected the cervical spine from abnormal posture during sustained full cervical flexion.

Furthermore, correlation analysis showed that CVA was significantly negative correlation with the muscle activity of Phase I. It has been reported that if changes in the neuromuscular system occur due to continuous FHP, moments of the neck during sustained cervical flexion increase cervical erector spinae and stabilize the neck. ${ }^{24}$ Increased Phase I in the FHP group is believed to be the result of a process that occurs during sustained full cervical flexion to protect the cervical spine from abnormal postures. It appears that increased activity observed in Phase I was the result of a motor control strategy used to provide cervical spine stability. However, correlation analysis failed to detect a significant correlation between CVA and FRR. FHP can be described as excessive forward positioning of the head relative to vertical and is usually quantified using CVA, where a low CVA value represents an unsuitable FHP. According to a previous study, a CVA $>48.7^{\circ}$ is defined as a "good" posture, and a CVA of $<43.8^{\circ}$ as a "bad" posture. However, since the FHP group in this study consisted of subjects at the "fair" $\left(43.8^{\circ} \leq \mathrm{CVA}<48.7^{\circ}\right)$ level, ${ }^{14}$ we considered this was why no significant correlation was found between FRR and CVA. We suggest additional studies be conducted on subjects with a wider range of CVA levels to determine whether FRR and CVA are correlated.

Several limitations of this study require consideration. First, only 19 healthy adults were recruited, and thus, which severely limits the generalizability of our findings. Second, FRR values probably depend on degree of muscle fatigue, further study is required to explore this topic. Third, phase-by-phase comparisons should be considered using percentage RVC, as the amount of electromyogram varies depending on the subject's char- acteristics.

In conclusion, a forward head posture increases cervical erector spinae activity during sustained cervical full flexion and reduces FRR, which can cause fatigue of cervical erector spinae muscles. Also, subjects with a smaller CVA, cervical muscle activity can be increased during sustained full cervical flexion and increase neck muscle tension.

\section{REFERENCES}

1. Koseki T, Kakizaki F, Hayashi S et al. Effect of forward head posture on thoracic shape and respiratory function. J Phys Ther Sci. 2019;31(1):638.

2. Fernández-de-las-Peñas C, Alonso-Blanco C, Cuadrado ML et al. Trigger points in the suboccipital muscles and forward head posture in tension-type headache. Headache. 2006;46(3):454-60.

3. Szeto GP, Straker L, Raine S. A field comparison of neck and shoulder postures in symptomatic and asymptomatic office workers. Appl Ergon. 2002;33(1):75-84.

4. Park JH, Kim JH, Kim JG et al. The effects of heavy smartphone use on the cervical angle, pain threshold of neck muscles and depression. Adv Sci Technol Lett. 2015;91(3):12-7.

5. Hansraj KK. Assessment of stresses in the cervical spine caused by posture and position of the head. Surg Technol Int. 2014;25(25):277-9.

6. Patwardhan AG, Khayatzadeh S, Havey RM et al. Cervical sagittal balance: A biomechanical perspective can help clinical practice. Eur Spine J. 2018;27(1):25-38.

7. Patwardhan AG, Havey RM, Muriuki M et al. Postural compensation and disc mechanics in forward head posture: A novel laboratory model of cervical sagittal balance. Spine J. 2013;13(9):S100.

8. Kwon JW, Son SM, Lee NK. Changes in upper-extremity muscle activities due to head position in subjects with a forward head posture and rounded shoulders. J Phys Ther. 2015;27(6):1739-42.

9. Pop MS, Mihancea P, Debucean D. Posture optimization-is it the key to myofascial neck pain relief? Arch Balk Medical Union. 2018;53(4);5739.

10. Murphy BA, Marshall PW, Taylor HH. The cervical flexion-relaxation ratio: Reproducibility and comparison between chronic neck pain patients and controls. Spine. 2010;35(24):2103-8.

11. Callaghan JP, Dunk NM. Examination of the flexion relaxation phenomenon in erector spinae muscles during short duration slumped sitting. Clin Biomech. 2002;17(5):353-60.

12. Alschuler KN, Neblett R, Wiggert E et al. Flexion-relaxation and clinical features associated with chronic low back pain: A comparison of different methods of quantifying flexion-relaxation. Clin J Pain. 2009;25 (9):760-6.

13. Colloca CJ, Hinrichs RN. The biomechanical and clinical significance of the lumbar erector spinae flexion-relaxation phenomenon: A review of literature. J Manipulative Physiol Ther. 2005;28(8):623-31.

14. Choi KH, Cho MU, Park CW et al. A comparison study of posture and fatigue of neck according to monitor types (moving and fixed monitor) by using flexion relaxation phenomenon (FRP) and craniovertebral an- 
gle (CVA). Int J Environ Res Public Health. 2020;17(17):6345.

15. Alessa FM, Ning X. Lumbar range of motion and flexion relaxation phenomenon onset during static trunk bending postures. Proc Hum Factors Ergon Soc Annu Meet. 2018;62:915-9.

16. Neblett R, Mayer TG, Brede E et al. Correcting abnormal flexion-relaxation in chronic lumbar pain: Responsiveness to a new biofeedback training protocol. Clin J Pain. 2010;26(5):403.

17. Neblett R, Brede E, Mayer TG et al. What is the best surface emg measure of lumbar flexion-relaxation for distinguishing chronic low back pain patients from pain-free controls? Clin J Pain. 2013;29(4):334.

18. Pialasse JP, Dubois JD, Choquette MHP et al. Kinematic and electromyographic parameters of the cervical flexion-relaxation phenomenon: The effect of trunk positioning. Ann Phys Rehabil Med. 2009;52(1):4958.

19. Yoo WG, Park SY, Lee MR. Relationship between active cervical range of motion and flexion-relaxation ratio in asymptomatic computer workers. J Physiol Anthropol. 2011;30(5):203-7.
20. Sommerich CM, Joines SM, Hermans V et al. Use of surface electromyography to estimate neck muscle activity. J Electromyogr Kinesiol. 2000;10(6):377-98.

21. Kim YH, Jung JH. Effects of pnf program on neck pain, cervical range of motion, pressure pain, and cervical flexion-relaxation ratio in vdt worker: A case study. PNF \& Mov. 2015;13(4):197-202.

22. Bonney RA, Corlett EN. Head posture and loading of the cervical spine. Appl Ergon. 2002;33(5):415-7.

23. Singla D, Veqar Z. Association between forward head, rounded shoulders, and increased thoracic kyphosis: A review of the literature. J Chiropr Med. 2017;16(3):220-9.

24. Mousavi-Khatir R, Talebian S, Maroufi N et al. Effect of static neck flexion in cervical flexion-relaxation phenomenon in healthy males and females. J Bodyw Mov Ther. 2016;20(2):235-42.

25. Indahl A, Kaigle AM, Reikerås $\mathrm{O}$ et al. Interaction between the porcine lumbar intervertebral disc, zygapophysial joints, and paraspinal muscles. Spine. 1997;22(24):2834-40. 